\title{
A novel Glycine soja homeodomain-leucine zipper (HD-Zip) I gene, Gshdz4, positively regulates bicarbonate tolerance and responds to osmotic stress in Arabidopsis
}

\author{
Lei Cao, Yang Yu, Huizi DuanMu, Chao Chen, Xiangbo Duan, Pinghui Zhu, Ranran Chen, Qiang Li,
} Yanming Zhu* and Xiaodong Ding ${ }^{*}$

\begin{abstract}
Background: Wild soybean (Glycine soja) is a highly adaptive plant species which can grow well in saline-alkaline soils. In soybean genome, there exist about 140 HD-Zip (Homeodomain-leucine Zipper) genes. HD-Zip transcription factor family is one of the largest plant specific superfamilies and plays important roles in response to abiotic stresses. Although HD-Zip transcription factors have been broadly reported to be involved in plant resistance to abiotic stresses like salt and drought, their roles in response to bicarbonate stress is largely unknown.

Results: From our previous transcriptome profile analysis of wild soybean treated by $50 \mathrm{mM} \mathrm{NaHCO}$, we identified an HD-Zip gene (Gshdz4) which showed high response to the alkaline stress. Our result of qRT-PCR showed that the expression of Gshdz4 was induced by alkaline stress $\left(\mathrm{NaHCO}_{3}\right)$ in both leaves and roots of wild soybean. Overexpression of Gshdz4 in Arabidopsis resulted in enhanced tolerance to $\mathrm{NaHCO}_{3}$ and $\mathrm{KHCO}_{3}$ during the process of plant growth and development. However, the growths of transgenic and WT plants were not significantly different on the medium with high pH adjusted by $\mathrm{KOH}$, implicating Gshdz4 is only responsible for resisting $\mathrm{HCO}_{3}{ }^{-}$but not high $\mathrm{pH}$. The transgenic plants had less MDA contents but higher POD activities and chlorophyll contents than the WT plants. Moreover, the transcript levels of stress-related genes, such as NADP-ME, $H^{+}$-Ppase, RD29B and KIN1 were increased with greater extent in the transgenic plants than the wild plants. On the contrary, Gshdz4 overexpression lines were much sensitive to osmotic stress at seed germination and stocking stages compared to the wild plants.
\end{abstract}

Conclusions: We revealed that the important and special roles of Gshdz4 in enhancing bicarbonate tolerance and responding to osmotic stress. It is the first time to elucidate these novel functions of HD-ZIP transcription factors. All the evidences broaden our understanding of functions of HD-Zip family and provide clues for uncovering the mechanisms of high tolerance of wild soybean to saline-alkaline stresses.

Keywords: Gshdz4, Transcription factor, Bicarbonate tolerance, Osmotic stress, Glycine soja, Arabidopsis

Abbreviations: ABA, Abscisic acid; CTRs, Carboxy-terminal regions; Gshdz4, Glycine soja homeodomain-leucine zipper 4; $\mathrm{H}^{+}$-Ppase, $\mathrm{H}^{+}$-pyrophosphatase; KIN1, Kinase 1; MDA, Malondialdehyde; NADP-ME, NADP-dependent malic enzyme; NLS, Nuclear localization signal; NTRs, Amino-terminal regions; P5CS, 1-Pyrroline-5-carboxylate synthetase; OX, Overexpression; POD, Peroxide; RD22, Responsive to dehydration 22; RD29A, Responsive to dehydration 29A; RD29B, Responsive to dehydration 29B; SD, Synthetically defined medium; USER, Uracil-specific excision reagent; WT, Wild type

\footnotetext{
* Correspondence: ymzhu2001@neau.edu.cn; xiaodong.ding@neau.edu.cn
} Key Laboratory of Agricultural Biological Functional Genes, Northeast Agricultural University, Harbin 150030, China 


\section{Background}

Adverse environmental factors, such as salt, alkali, and/ or drought stresses greatly limit the growth and development of plants which are sessile on the ground. The soil salinity-alkalinity causes crop yield reduction which has become a serious problem for the securities of food supplies [1]. Plants have evolved with complex molecular mechanisms to survive and to keep normal growth under stress conditions. As a result of RNA sequencing profiles of wild soybean roots which were exposed to sodium bicarbonate $\left(\mathrm{NaHCO}_{3}\right)$ [2], a couple of transcription factor genes like HD-ZIP [3], MYB [4], WRKY [5], NAC [6], bZIP [7], C2H2 [8] and TIFY [9] were identified as alkali stress-responsive genes. Although lots of the previous studies have been reported about the roles of transcription factors in plant tolerance to salt or drought stress, little is known regarding their roles in alkali stress. Therefore, a deep comprehending of the essential mechanisms of plant responses to alkali stress is urgently needed and will contribute greatly to cultivating alkaline-tolerant crop varieties by biotechnology.

In our previous RNA sequencing study [9], we identified an HD-Zip gene from the wild soybean transcriptome. This gene is highly homologous with Gmhdz4 of cultivated soybean (G. $\max$ ) and encodes a putative homeodomain-leucine zipper protein. The HD-Zip family is unique to the plant kingdom [3]. In a previous study, Chen et al. identified 140 HD-Zip family genes from G. $\max$ genome and this family can be phylogenetically classified into I, II, III and IV subfamilies [10]. HDZip proteins have a conserved homeodomain followed by a leucine zipper in most members or a MEKHLA domain only in subgroup IV members [11]. Homeodomain is a kind of DNA binding domain involved in the transcriptional regulation of key eukaryotic developmental processes and it may bind to DNA as monomers or as homo- and/or heterodimers in a sequence-specific manner. Leucine zipper is consist of respective amino acid sequences on an idealized alpha helix revealed by a periodic repetition of leucine residues at every seventh position over a distance covering eight helical turns [12].

The HD-Zip protein family has been found in a wide variety of plant species, and many efforts have been undertaken to evaluate the functions of HD-Zip genes. HD-Zip I proteins mainly participate in responses to abiotic stresses and the regulation of organ growth and developmental process [3]. For example, the expression of Oshox 22 was strongly induced by salt stress, abscisic acid (ABA), and polyethylene glycol treatment (PEG), and weakly by cold stress in rice [13]. ATHB7 and $A T H B 12$ were both strongly induced by abscisic acid (ABA) and water-deficit, and functioned as negative primary regulators of ABA response in Arabidopsis [14]. Zmhdz10, positively regulated drought and salt tolerance in both rice and Arabidopsis [15]. HB1 from HD-Zip subfamily I of Medicago truncatula, was strongly induced by salt stress in root apices and played as regulator of root architecture and lateral root emergence [16]. However, there is little information about HD-Zip I genes responding to alkaline stress.

Bicarbonate stress, including adverse effects of $\mathrm{Na}^{+}, \mathrm{CO}_{3}^{2-}$, $\mathrm{HCO}_{3}^{-}$and high $\mathrm{pH}$, also elicits negative impacts on plant growth and development. Excessive ion of organic acids such as malate, succinate and citrate were accumulated under bicarbonate $\left(\mathrm{NaHCO}_{3}\right)$ stress, leading to inhibit growth of new root and shoot [17]. The oxidation/reduction-related genes also participate actively in reacting to environmental stimulation which always reflect on physiological indexes such as MDA content and POD activity. Chlorophyll content is also a standard to assess damage of basic cell structure in green plants under stress treatment.

In this study, we explored the functions of Gshdz4 under alkaline and osmotic stresses. The data showed that there may be different molecular mechanisms and pathways between alkaline stress and osmotic stress and Gshdz4 functioned as a regulator of bicarbonate stress, providing new evidences to fully understand the functions of HD-Zip genes.

\section{Results}

\section{Identification and bioinformatics analysis of Gshdz4}

In our preceding work [2], we noticed that one wild soybean gene which is highly homologous with Gmhdz4 gene of cultivated soybean was induced by alkaline stress. We designed this gene as Gshdz4. Evolution analysis implied that Gshdz4 belongs to a conserved $\delta$ subgroup of HD-Zip I subfamily [10, 18]. Since Gshdz4 was more significantly up-regulated than the other HD-Zip I members in transcriptome profiling analysis of Glycine soja roots treated by alkali stress [2], it was chosen as a further research object in term of functional analyses in this study.

The full-length cDNA of Gshdz4 was isolated from Glycine soja by using homologous cloning strategy. Sequence analysis confirmed that Gshdz4 contains an open reading frame (ORF) of 648-bp that encodes a protein of 215 amino acids with an estimated molecular weight of 25118.2 Da and a theoretical pI of 6.48. Gshdz4 protein has a conserved homeodomain (aa57-113) and leucinezipper domains (aa132-182) (http://www.ncbi.nlm.nih.gov/ Structure/cdd/wrpsb.cgi) (Fig. 1b). The cDNA sequence of Gshdz4 shares high identity to several gene CDS sequences downloaded from the genome database of Glycine max in Phytozome (http://phytozome.jgi.doe.gov/pz/ portal.html), the green plants genomic database.

A BLASTP search at NCBI showed that Gshdz4 shared $66 \%$ sequence identity with Glycine max Gmhdz10 


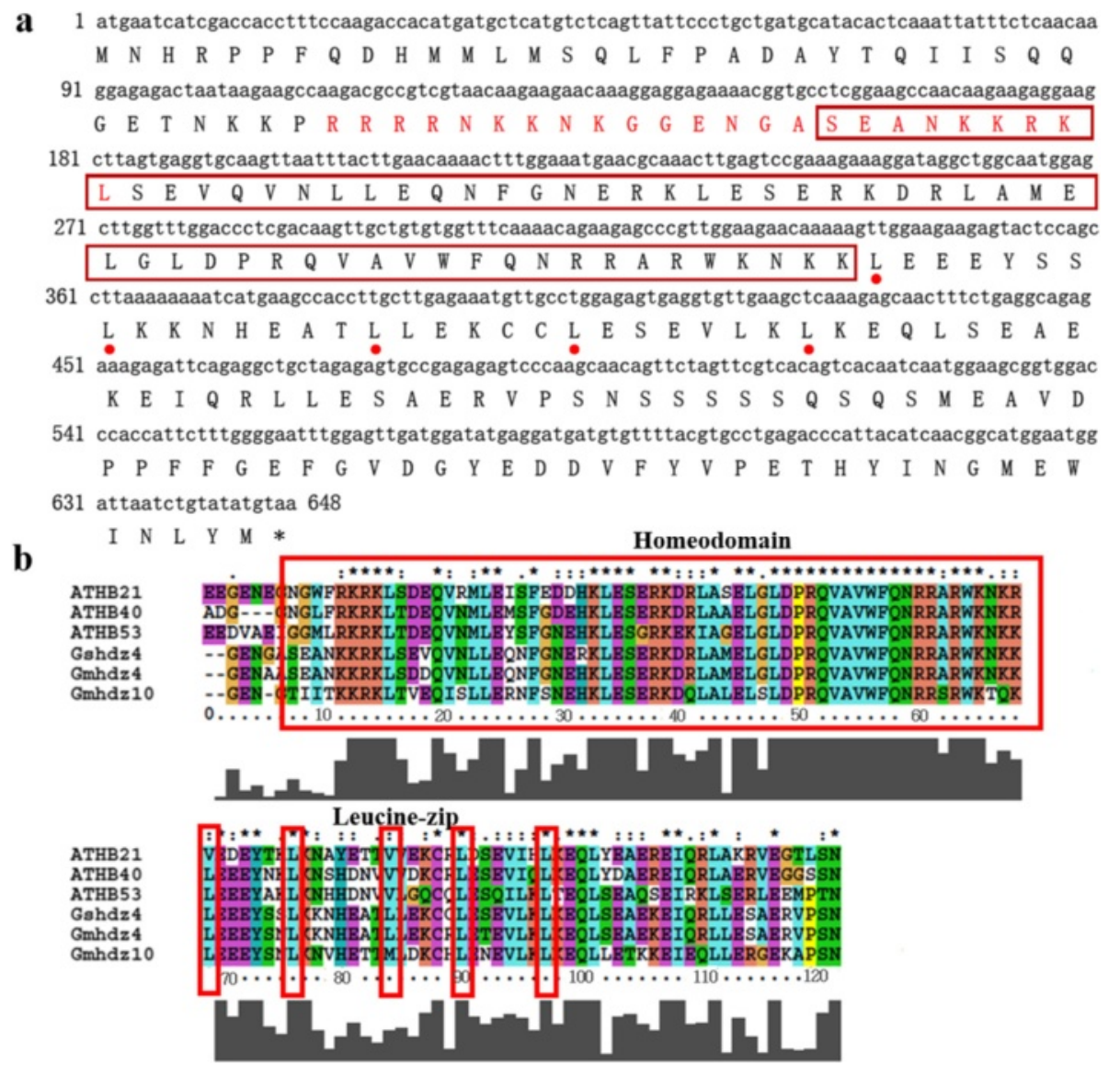

Fig. 1 Sequence analysis of Gshdz4. a Gshdz4 nucleotide and its deduced amino acid sequences. The homeodomain (HD) and leucine-zip (ZIP) motifs are indicated by red frame and red points, respectively. One putative nuclear localization signal (NLS) sequence is marked in red. b Sequence alignment of HD domains of soybean and Arabidopsis subgroup $\delta$ members. The conserved leucine residues are indicated by red frames

(Glyma02g06560) and $64.7 \%, 58.6 \%, 66.5 \%$ with Arabidopsis thaliana ATHB21 (AT2G18550), ATHB40 (AT4G 36740), ATHB53 (AT5G66700), respectively. One putative NLS (nuclear localization signal) motif was predicted to be located in the N-terminus by an online software Predict Protein (https://www.predictprotein.org/) (Fig. 1a).

\section{Spatial and temporal expression patterns of Gshdz4}

In order to investigate the induced expression patterns of Gshdz4 in the roots of wild soybean under $50 \mathrm{mM}$ $\mathrm{NaHCO}_{3}$ stress, we carried out qPCR (quantitative realtime PCR) analyses. Under normal condition, the expression of Gshdz4 kept on a relatively stable level during the whole day and no peak was found (Fig. 2a). However, its transcript level in roots was significantly up-regulated by alkaline stress in a relatively early period of $6 \mathrm{~h}$ after treatment (Fig. 2a).

Nevertheless, the expression of Gshdz4 in leaves was also induced by alkaline stress and reached a peak at $1 \mathrm{~h}$, but reduced to valley at $12 \mathrm{~h}$ after treatment
(Fig. 2b). It is remarkable that the induced expression level of Gshdz4 in roots was dramatically higher than that in leaves. The possible reasons for the strong response in roots might be that the plant roots are the exact region of stress perception and the organ directly exposed to stress damage, or the responsive mechanisms for Gshdz4 expression in roots may differ to that in leaves [19]. Whatever, these results suggest that Gshdz4 is an alkaline-inducible gene and plays important roles in response toNaHCO 3 stress in wild soybean.

In addition, the spatial expression of Gshdz4 was also verified in eight main tissues using qPCR. Gshdz4 was differentially expressed in all of the tested wild soybean tissues and organs, including young and mature leaves, young and mature stems, epicotyls, hypocotyls, root tips and flowers (Fig. 2c). The results indicated that, among the eight tissues examined in this study, Gshdz4 exhibited the highest expression level in roots, consistent with its important role of stress resistance in roots. On the contrary, the Gshdz4 transcript level in leaves especially 
$\mathbf{a}$

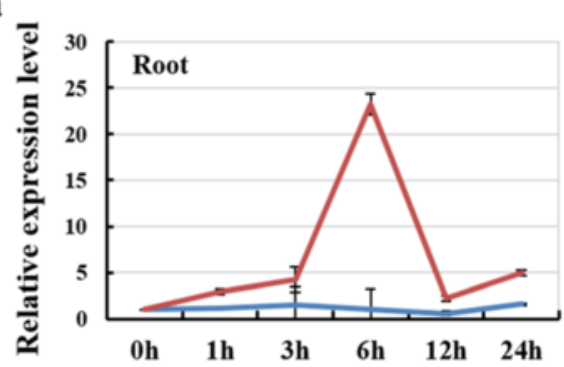

b

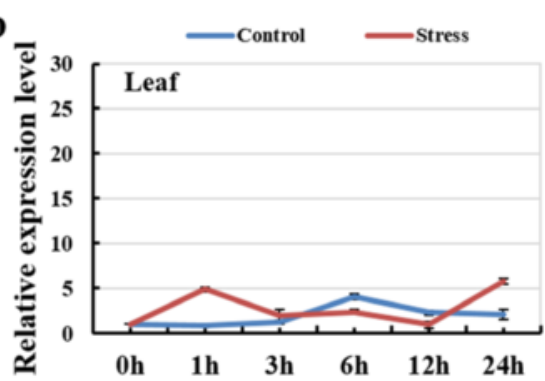

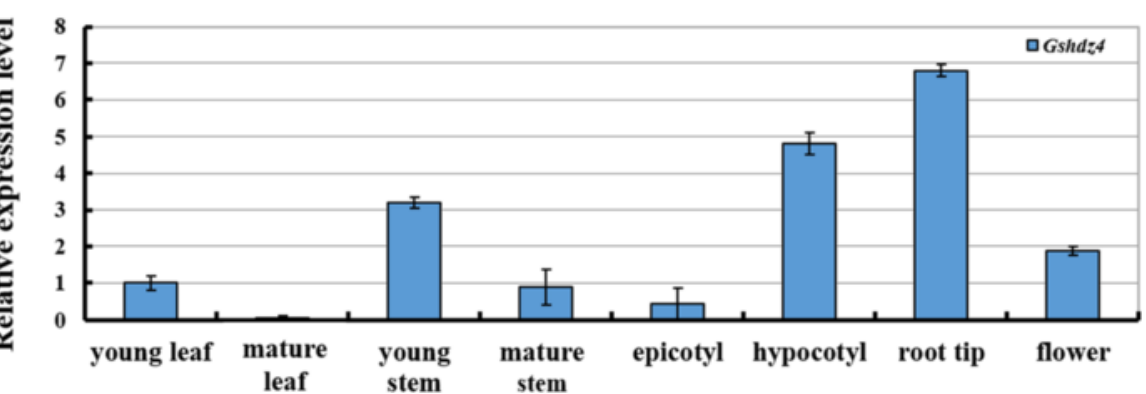

Fig. 2 Expression and functional analysis of Gshdz4. a and $\mathbf{b}$ Gshdz4 expression analysis (RT-qPCR) of roots and leaves of 3-week wild soybean seedlings which were subjected to $50 \mathrm{mM} \mathrm{NaHCO}_{3}$ treatment. c Expression patterns of Gshdz4 in various tissues of wild soybean. Values represent means of three biological replicates; error bars indicate SD

in old ones was relatively lower, implicating that the diverse mechanisms and roles of Gshdz4 in roots and leaves [19].

\section{Gshdz4 is a nucleus-localized protein}

Sequence analysis showed that Gshdz4 has one putative NLS motif at the N-terminus and illustrated that Gshdz4 may target to the nucleus. To affirm the prediction, the pBSK-Gshdz4-eGFP construct was used to investigate the subcellular localization of Gshdz4 protein in onion epidermal cells through biolistic bombardment. The figures showed that the GFP signal was detected in the nuclei of the onion cells by pBSK-Gshdz4-eGFP construct (Fig. 3). As the control, the eGFP signal produced by pBSK-eGFP construct was found throughout the whole cells (Fig. 3). These results suggest that Gshdz4 should be a nucleus-localized protein which is one of the basic characteristics of transcription factors.

Gshdz4 lacks transcription activation activity in yeast cells To exam whether Gshdz4 performs trans-activation activity, Gshdz4 were fused to a DNA binding domain (GAL4), and its ability to activate transcription of $\mathrm{LacZ}$ and HIS3 reporter genes was determined. The AH109 yeast strain was transformed with either pGBKT7GsDREB (positive control), or pGBKT7 (negative control), pGBKT7-Gshdz4,. The AH109 yeast cells carrying one of the above plasmids grew equally well on $\mathrm{SD} /$-Trp medium containing X-gal. However, the positive control performed high galactosidase activity (blue colonies),

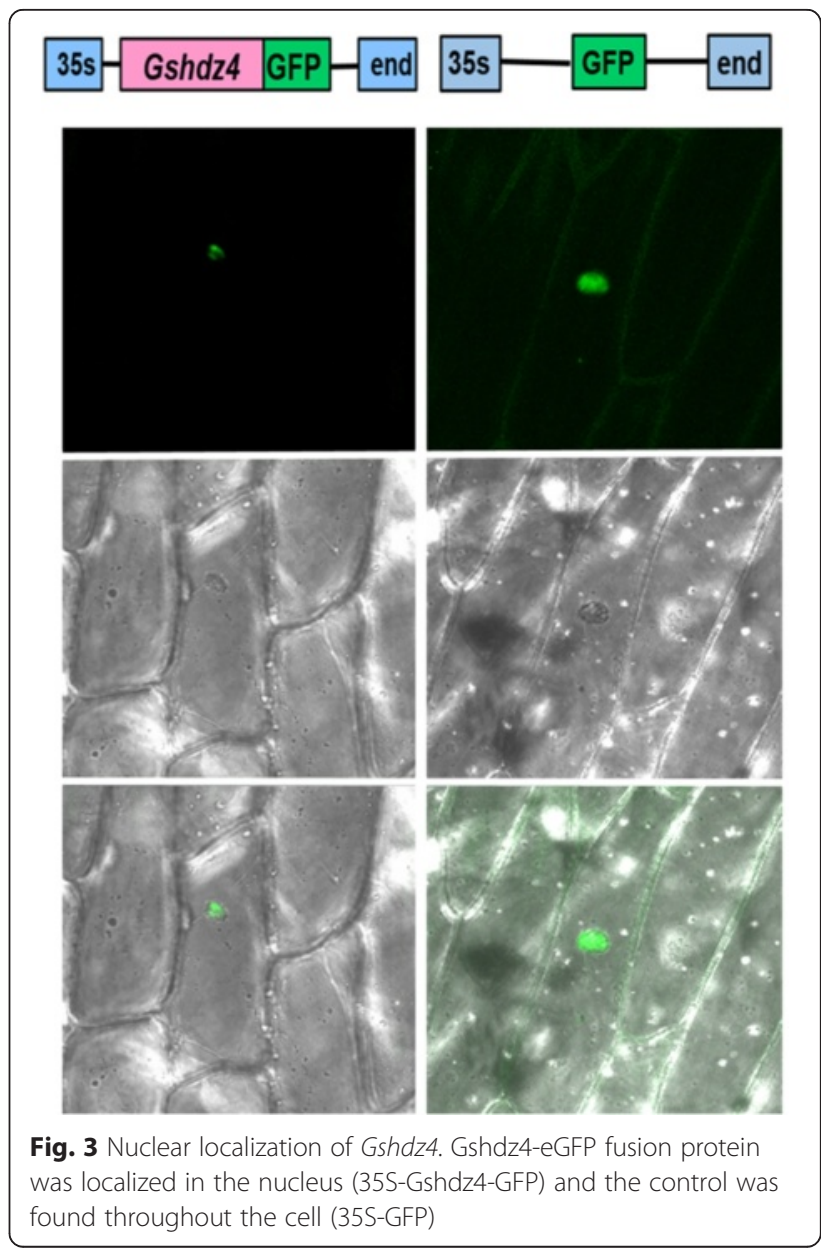


nevertheless the cells transformed with pGBKT7-Gsh $\mathrm{dz} 4$, or the negative control, exhibited no galactosidase activity (white colonies) (Fig. 4b). At the same time, the yeast cells carrying the positive control plasmid pGBKT7-GsDREB grew well on SD/-Trp-His medium but the cells with pGBKT7 or pGBKT7-Gshdz4 did not, indicating that Gshdz4 could not activate the reporter genes expression in yeast cells. It suggested Gshdz4 may be a transcription repressor or co-activator requires the other plant transcription components.

\section{Overexpression of $\mathrm{Gshdz} 4$ enhanced tolerance to $\mathrm{HCO}_{3}{ }^{-}$,} but not to $\mathrm{OH}^{-}$

In order to assess the function of Gshdz4 in alkali stress, Gshdz4 overexpression lines of Arabidopsis were generated. As a result, the Gshdz4 transgenic plants had similar germination rates with the wild type, although their germination rates showed a little bit of fluctuation in the first 3 days after imbibition (Additional file 1: Figure S1). There was no difference found in growth between the overexpression lines and WT plants in the normal condition (Additional file 2: Figure S3). And then, we compared the germinations and growths of transgenic lines with WT on $1 / 2$ MS medium supplemented with $6 \mathrm{mM}$ or $7 \mathrm{mM} \mathrm{NaHCO}$. Under $\mathrm{NaHCO}_{3}$ treatment, the seeds from both Gshdz4 overexpression lines and WT were capable of developing healthy cotyledons following seed coat breakage and radicle emergence (Fig. 5b) and the germination rate data showed no difference (Additional file 1: Figure S1). However, after 8 days on the medium containing $6 \mathrm{mM}$ or $7 \mathrm{mM} \mathrm{NaHCO}$, the survival rates of transgenic lines were significantly higher than those of WT. The WT plants showed sensitivity to $\mathrm{NaHCO}_{3}$ treatments. In addition, on the 20th day after germination, the transgenic lines possessed much higher average percentage of seedlings with open and green leaves (> four green leaves) than WT, and most seedlings of WT turned white and gradually died (Fig. 5b). However, among the three transgenic lines (\#14, \#20 and \#23), \#23 line demonstrated the higher survival rate and percentage of green leaves than \#20 and \#14. These results indicate that overexpression of

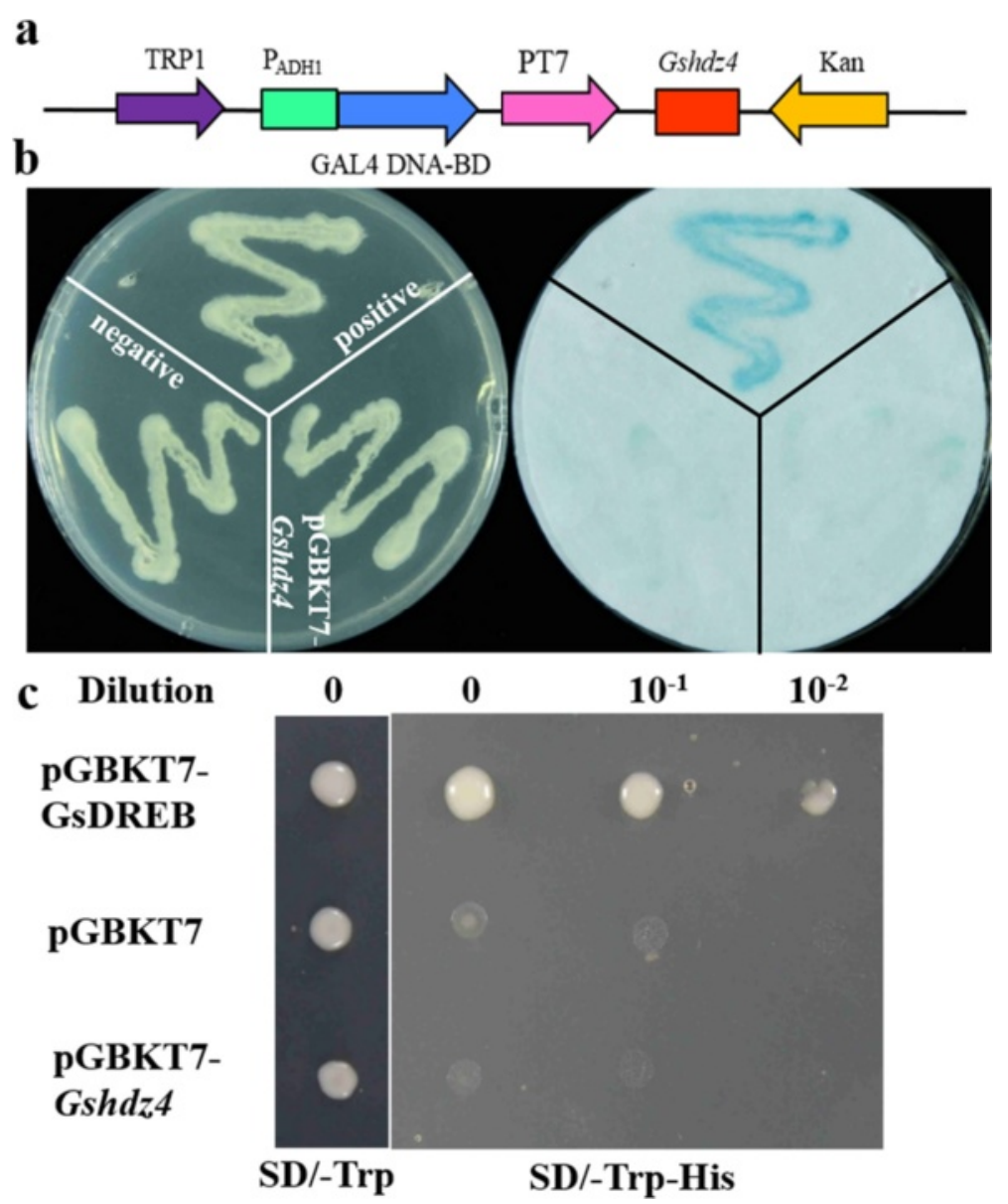

Fig. 4 Transcription activity analysis of Gshdz4. a The construct of pGBKT7-Gshdz4. b Galactosidase (LacZ) assay. c His3 reporter assay 

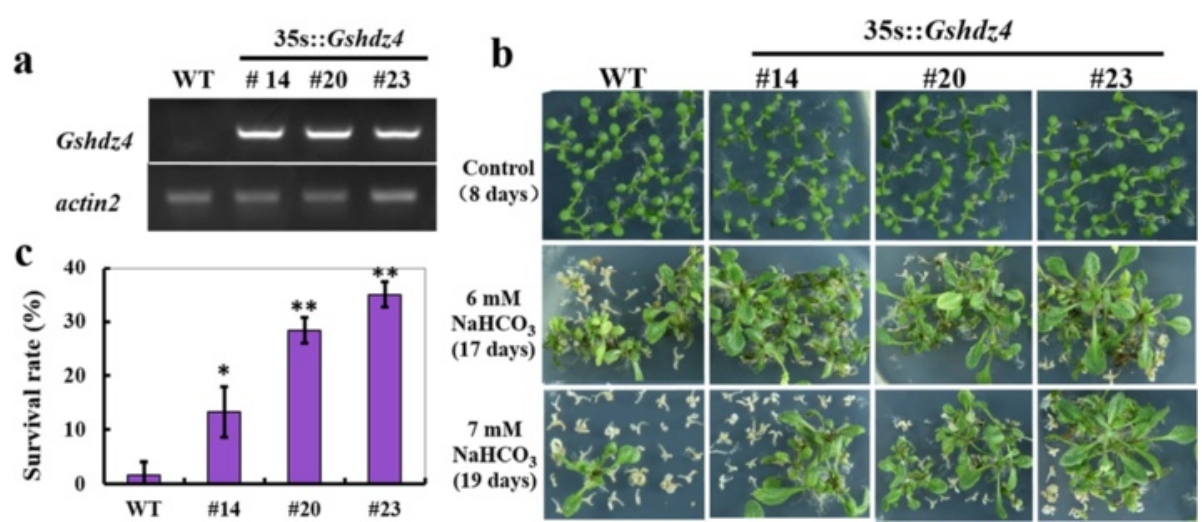

Fig. 5 Overexpression of Gshdz4 enhanced tolerance to $\mathrm{NaHCO}_{3}$ stress in germination stage. a Transcription levels of Gshdz4 in transgenic Arabidopsis (\#14, \#20 and \#23) and WT by RT-PCR. b NaHCO 3 stress tolerance of Gshdz4 transgenic Arabidopsis (\#14, \#20 and \#23). Plant growth under normal condition and under $\mathrm{NaHCO}_{3}$ stress $\left(0,6\right.$ or $7 \mathrm{mM} \mathrm{NaHCO}$ ). c Plant survival rates under $\mathrm{NaHCO}_{3}$ stress $(0$ or $6 \mathrm{mM} \mathrm{NaHCO}$ ). Values represent means of three biological replicates; error bars indicate SD. Significant differences are denoted with one or two stars if $P<0.05$ or $P<0.01$, by Student's $t$-test

Gshdz4 may reduce the damage of high alkaline stress on chlorophyll degradation and may enhance tolerance to $\mathrm{NaHCO}_{3}$ to maintain plant growth and development.

Gshdz4. OX also enhanced plant tolerance to $\mathrm{NaHCO}_{3}$ stress at the seedling stage. 7 days after germinating, the seedlings of Gshdz4 OX and WT were transferred onto 1/2 MS medium supplemented with $0 \mathrm{mM}, 6 \mathrm{mM}$ or $8 \mathrm{mM} \mathrm{NaHCO}_{3}$. The result showed that the growth and development of the WT plants were severely inhibited compared with those of Gshdz4 OX plants under $\mathrm{NaHCO}_{3}$ treatments (Fig. 6a). Gshdz4 OX lines had longer primary roots and more total weight than WT under alkaline stress (Fig. 6b, c), implying that Gshdz4 may play an important role in root development and water retention to enhance plants tolerance to $\mathrm{NaHCO}_{3}$ stress.

We further investigated the tolerance of Gshdz4 OX lines and WT to $\mathrm{NaHCO}_{3}$ stress at stocking stage. The 3-week-old soil-grown plants were irrigated with $125 \mathrm{mM} \mathrm{NaHCO}$ every 3 days for 2 weeks. The survey data showed that the transgenic plants demonstrated better stress tolerance to $\mathrm{NaHCO}_{3}$ than the WT (Fig. 6d). Under normal condition, almost no difference was found in plant growth, the contents of total chlorophyll and malondialdehyde (MDA), and POD activity of the WT and three transgenic lines. However, in the present of $125 \mathrm{mM} \mathrm{NaHCO}$, the total chlorophyll contents decreased in WT more than transgenic plants (Fig. 6e). These results give evidence of that Gshdz4 OX reduces the damage of high alkaline stress on plants and alleviated chlorophyll degradation. As an indicator of oxidative damage, the content of MDA generated during peroxidation of membrane lipids is often used under abiotic stresses $[20,21]$. Thus, the MDA contents were measured in the transgenic and WT plants under alkaline stress conditions and found that the WT accumulated obviously higher levels of MDA than Gshdz4 OX lines (Fig. 6f). Since the formation of reactive oxygen species (ROS) in the cells can be promoted by most abiotic stresses and subsequently hurts the plants [22, 23], we postulate that overexpression of Gshdz4 can probably inhibit the production of ROS and/or can even clean the oxidative products to protect plants from membrane damage. To confirm this observation, we determined the endogenous POD activity. The data showed that POD activity of each Gshdz4 OX line was higher than that of WT (Fig. 6g), meaning that transgenic plants have more ability to get rid of ROS products and extra free radicals to protect cell structure from damage.

In the alkaline soil, it is $\mathrm{HCO}_{3}^{-}$and/or $\mathrm{OH}^{-}$-rich environment. For the sake of further investigating if $\mathrm{HCO}_{3}^{-}$or $\mathrm{OH}^{-}$or both can generate stress to the plants, the Gshdz4 OX and WT plants were grown on the medium with $6 \mathrm{mM} \mathrm{KHCO}_{3}$ or the medium with $\mathrm{pH} 7.5$ and pH8.2 adjusted by $\mathrm{KOH}$. The results showed that all transgenic and WT plants had normal and similar growth on the medium with $\mathrm{pH} 7.5$ and $\mathrm{pH} 8.2$ adjusted by $\mathrm{KOH}$. However, on the medium supplemented with $6 \mathrm{mM} \mathrm{KHCO}_{3}$, the growths of both WT and transgenic lines were greatly inhibited, whereas the transgenic plants had a little higher survival rates than the WT (Fig. 7a). Furthermore, we observed that Gshdz4 OX and WT seeds had similar germination rates on $1 / 2$ MS medium or $1 / 2$ MS medium supplemented with $6 \mathrm{mM}$ $\mathrm{KHCO}_{3}$ or $\mathrm{KOH}$ which was used to adjust the medium to $\mathrm{pH} 7.5$ and 8.2 , although the germination were delayed to some extent on the medium with $6 \mathrm{mM}$ $\mathrm{KHCO}_{3}$ and the medium with pH8.2 (Additional file 3: Figure S2). To further investigate the effect of $\mathrm{HCO}_{3}{ }^{-}$or $\mathrm{OH}^{-}$on plant survival and root growth, we grew the 7day seedlings for 10 days onto the medium containing $8 \mathrm{mM} \mathrm{KHCO}_{3}$ or the medium with $\mathrm{pH} 5.8, \mathrm{pH} 7.5$ or pH8.2 adjusted by $\mathrm{KOH}$. The data indicated that the OX 


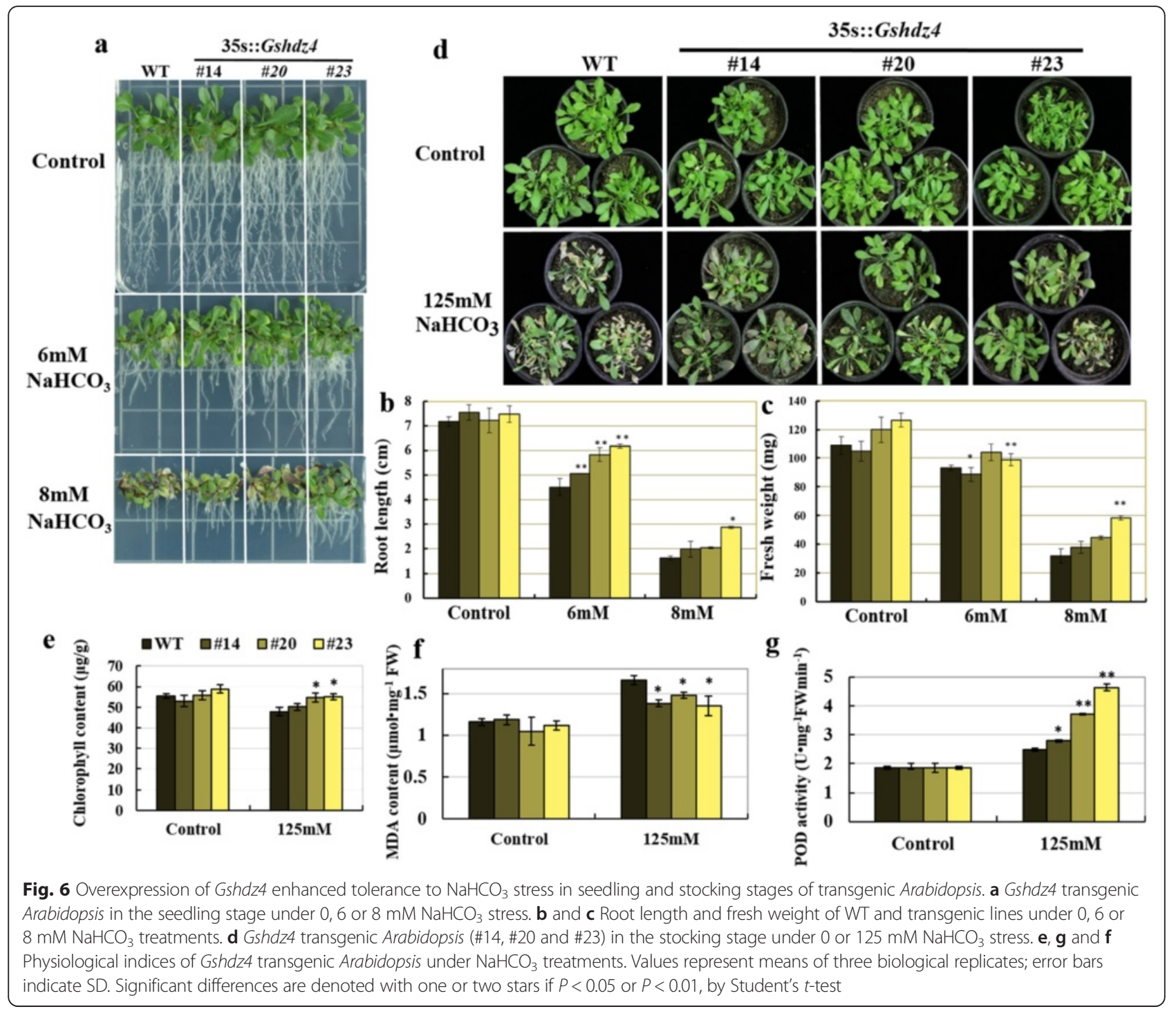

and WT plants had similar survival rate and root growth on normal 1/2 MS medium with pH5.8, pH7.5 or pH8.2 but the OX plants had higher survival rate and much better root growth than the WT on the medium containing 6 or $8 \mathrm{mM} \mathrm{KHCO}$ (Fig. 7b, d), implicating that Gshdz4 overexpression can promote plant tolerance to $\mathrm{HCO}_{3}^{-}$, but not $\mathrm{OH}^{-}$.

\section{Gshdz4 regulated the expression of the stress-relative} genes under $\mathrm{NaHCO}_{3}$ stress

The alkaline-resistant phenotypes of the Gshdz4 OX plants indicate that the expression of the stress response genes might be changed in the Gshdz4 OX lines. To prove this possibility, we compared the expression levels of the representative stress-inducible genes (NADP-ME, $H^{+}$-Ppase, RD29B and KIN1) in WT and Gshdz4 OX plants under abiotic stress. In the presence of $\mathrm{NaHCO}_{3}$ stress, these marker genes demonstrated much higher expression levels in the transgenic plants than the WT plants (Fig. 8a, b, c and d), confirming that Gshdz4 positively regulates the resistance to $\mathrm{NaHCO}_{3}$ stress in plants.

\section{Overexpression of Gshdz4 decreased tolerance to osmotic stress in transgenic Arabidopsis}

Since there is some evidence for cross-talk between signaling pathways which regulate different responses to alkaline and drought stresses [24, 25], we investigated and compared the drought-tolerant phenotypes of the Gshdz4 OX lines and WT plants. Under $325 \mathrm{mM}$ mannitol stress, the seeds of Gshdz4 OX lines had a slower pace of seed coat breakage and radical emergence than those of WT (Fig. 9a, b, c, d). In additionally, the seeds of Gshdz4 overexpression lines were unable to develop healthy cotyledons after breakage of seed coats, especially \#20 and \#23 (Fig. 9a). The overexpression lines exhibited less open leaves as well as green leaves than 


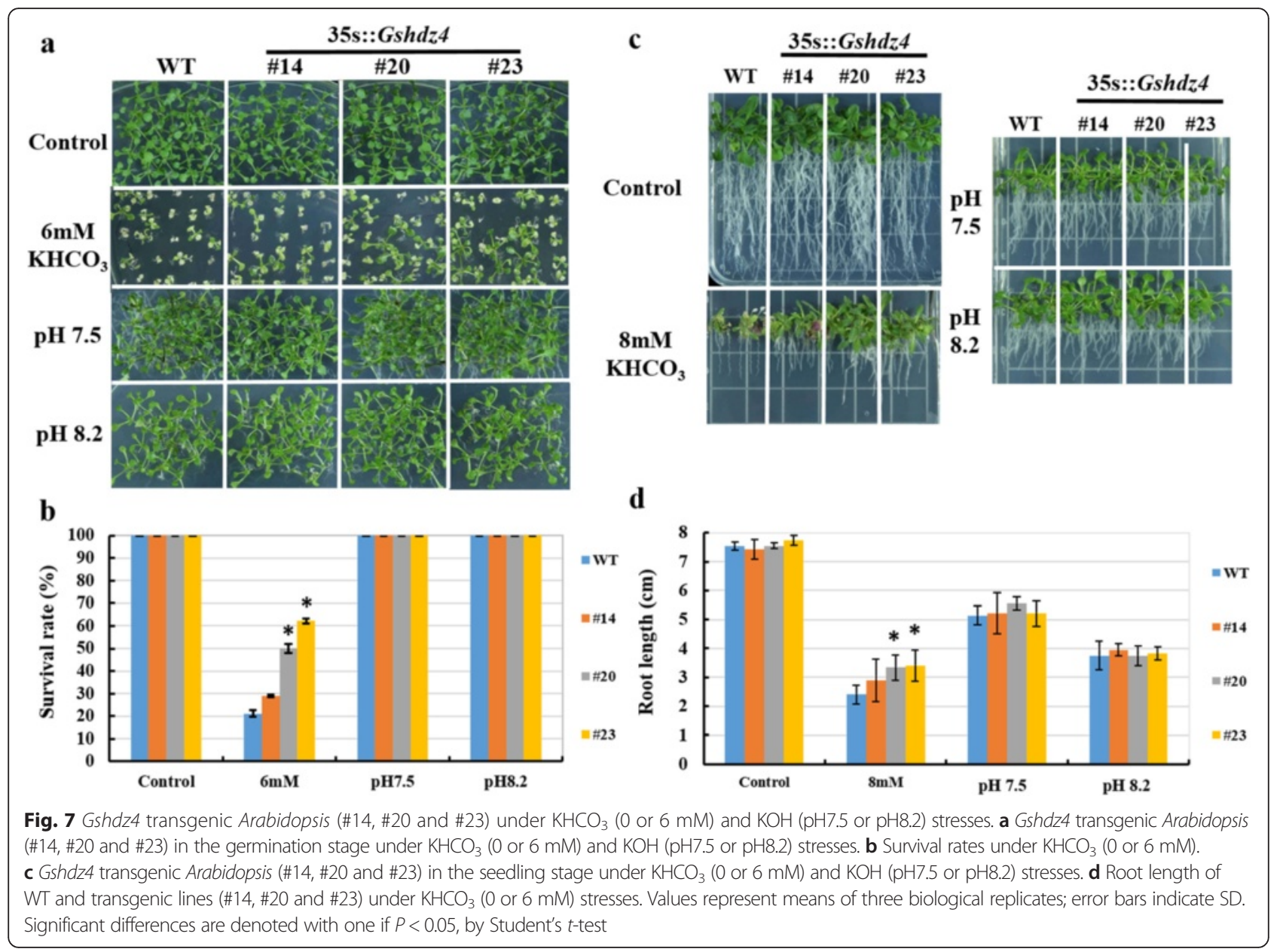

the WT at the seedling stage (Fig. 9a). At the adult stage of the WT and transgenic plants, all plants were stopped watering for 10 days to make the soil dry entirely. As shown in Fig. 9e, compared to the WT plants, Gshdz4 OX plants became severely wilted and impaired after dehydration stress. Together, the data indicate that overexpression of Gshdz4 enhanced drought sensitivity.

To further confirm our observation that Gshdz4 plays a negative role in plant drought-resistant signaling pathway, we compared the relative expression of osmotic stress response genes (RD29A, RD22and P5CS) in the WT and Gshdz4 OX plants. The result showed that there was no significant difference between WT and transgenic lines in relative expression levels of these genes, suggesting that Gshdz4 may negatively regulate plant drought-resistance through the other pathways.

\section{Discussion}

The saline and alkaline stresses are the main environmental stimulation limiting crop growth and leading to crop productivity reduction around the world [26]. Thus, understanding the mechanisms of the plant responses to alkaline or bicarbonate stress, and excavating bicarbonate-resistant genes, will promote biotechnological efforts to cultivate crop plants with enhanced resistant to bicarbonate-rich conditions [9]. Due to its strong resistance to alkaline and salt stresses, wild soybean is an ideal model for the research of the molecular mechanisms of bicarbonate and salt tolerances. A couple of transcription factors were found to be inducible in the transcriptome profiles of wild soybean roots which were treated with alkaline stress specifically. Among these transcription factors, Gshdz4 is one of highly inducible genes by alkaline stress [2]. They were proved to be diversely expressed in the process of alkaline stress response based on transcriptome profile analysis.

Gshdz4 belongs to HD-Zip protein family. The name is assigned based on its high homology with the sequences of G. max reported in previous study [10]. HD-ZIP family has four subgroups (I, II, III and IV). In Arabidopsis, HDZIP I genes play a part in response to abiotic stresses, ABA, blue-light and de-etiolation [3]. HD-ZIP II genes respond to illumination conditions [27], shade avoidance [28-30] and auxins [31, 32]. Moreover, several HD-ZIP I subfamily members, like ATHB7 and ATHB12 [14], Hahb- 

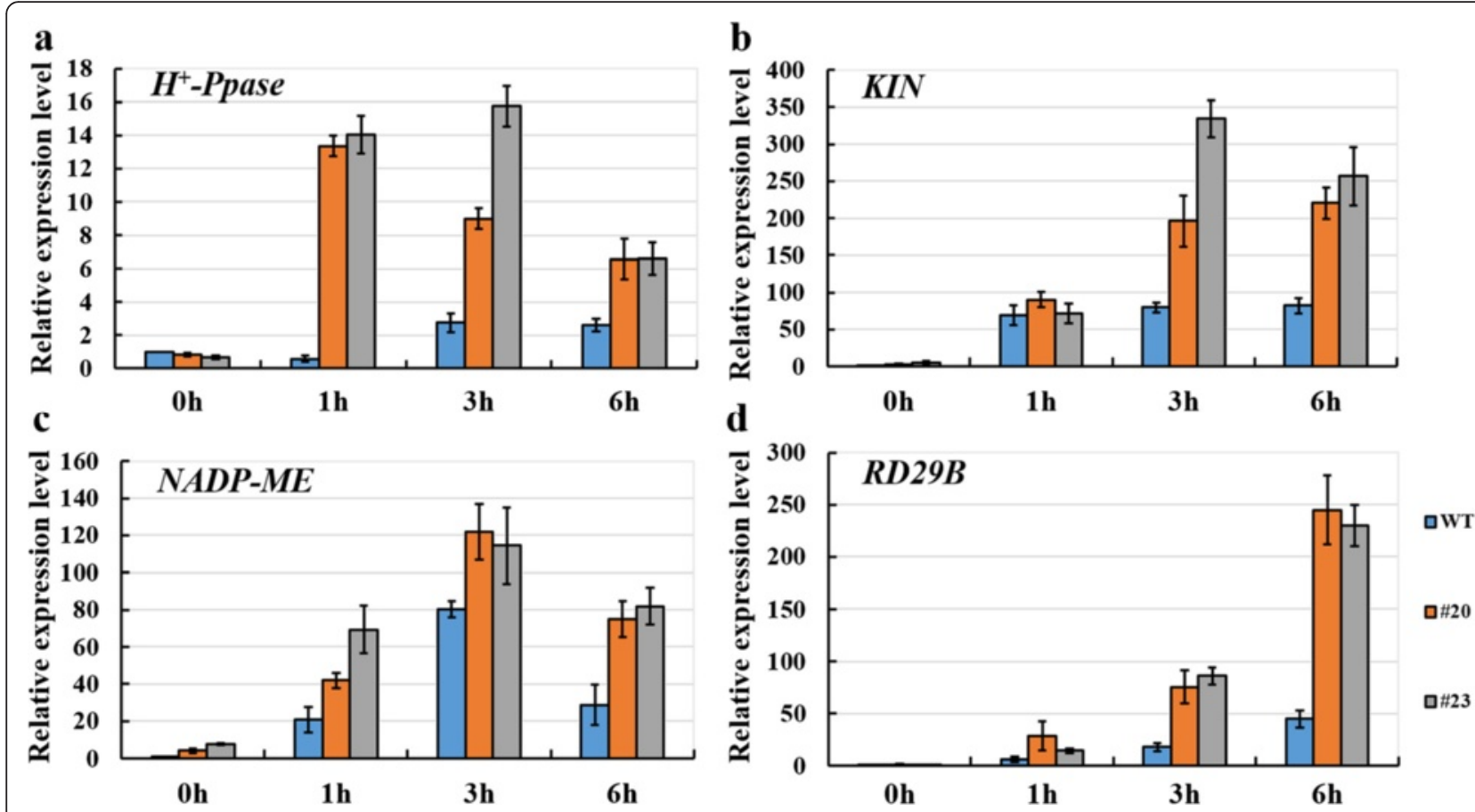

Fig. 8 Relative expression levels of alkaline stress-responsive genes in transgenic Arabidopsis plants (\#20 and \#23) under 50 mM NaHCO 3 stress. a, b, $\mathbf{c}$ and $\mathbf{d}$ showed the expression of $\mathrm{H}^{+}$-Ppase, KIN, NADP-ME, RD29B respectively. Values represent means of three biological replicates; error bars indicate SD

4 [33], MtHB1 [16], Oshox22 [13] and Zmhdz10 [15, 34], were involved chiefly in plant responses to abiotic stresses. Whereas, no any HD-Zip I proteins from Glycine soja have been verified and their physiological and biological functions have still unknown until now. In our study, a novel HD-Zip I protein from subgroup $\delta$, Gshdz4, was first identified from Glycine soja and then was functionally characterized for its response and tolerance to drought and alkaline stress in transgenic Arabidopsis plants.

As it was shown in Fig. 1a, the amino acids in red frame were homeodomain and the following leucine residues marked with red points were leucine-zip domain. And the amino acids in red were NLS sequences predicted by the online software. So Gshdz4 has basic structures similar to homologs from Glycine max and Arabidopsis and may locate in nucleus as common transcription factors. Sequence analysis affirmed that Gshdz4 shared high structural homology with Gmhdz4, a soybean HD-Zip protein. The results of spatial and temporal expression patterns suggested that Gshdz4 was significantly induced by $\mathrm{NaHCO}_{3}$ stress treatment, espposecially in roots (Fig. 2a) but not in leaves (Fig. 2b). It is possible that the plant roots are the major signal perception organ of soil stresses, which induces Gshdz4 to express in roots but not in leaves. In analysis of tissue-specific expression patterns, we found that Gshdz4 had higher levels in young stem, hypocotyl and especially in root tip than the other organs of wild soybean.
The transient expression and yeast assays of Gshdz4 showed that the full-length gene could not activate any reporter genes, giving the possibility that Gshdz4 may act as a repressor, or a co-activator requiring additional factors to fulfill its function. In cotton, GhHOX3 is an HD-Zip family gene and is related with cotton fiber elongation and contributes to promoting cell expansion during leaf growth $[35,36]$. GhSLR1 interferes with the GhHOX3-GhHD1 interaction and represses target gene transcription [35]. In Arabidopsis, the transcriptional repression of BODENLOS is controlled by an HD-Zip transcription factor, HB5 [37]. On the other hand, maize HD-Zip, Zmhdz10, acts as transcriptional activators [15].

In this study, we first characterized a wild soybean HD-Zip I subfamily gene, Gshdz4, which can enhance plant tolerance to $\mathrm{HCO}_{3}{ }^{-}$, but not $\mathrm{OH}^{-}$(high $\mathrm{pH}$ ). As shown in the phenotypic data, the transgenic Arabidopsis lines $(\# 14, \# 20, \# 23)$ showed significant tolerance to $\mathrm{NaHCO}_{3}$ at the germination, young seedling and mature seedling stages. Although there was no difference on germination rates among all lines, the transgenic seedlings possessed higher survival rate, greater root length and heavier fresh weight than the WT, suggesting that overexpression of Gshdz4 enhanced plant tolerance to alkaline stress. The current knowledge tells us that the alkaline stress in soil is mainly caused by high concentration of $\mathrm{NaHCO}_{3}$ [26]. Our data showed that overexpression of Gshdz4 resulted in enhanced plant tolerance 


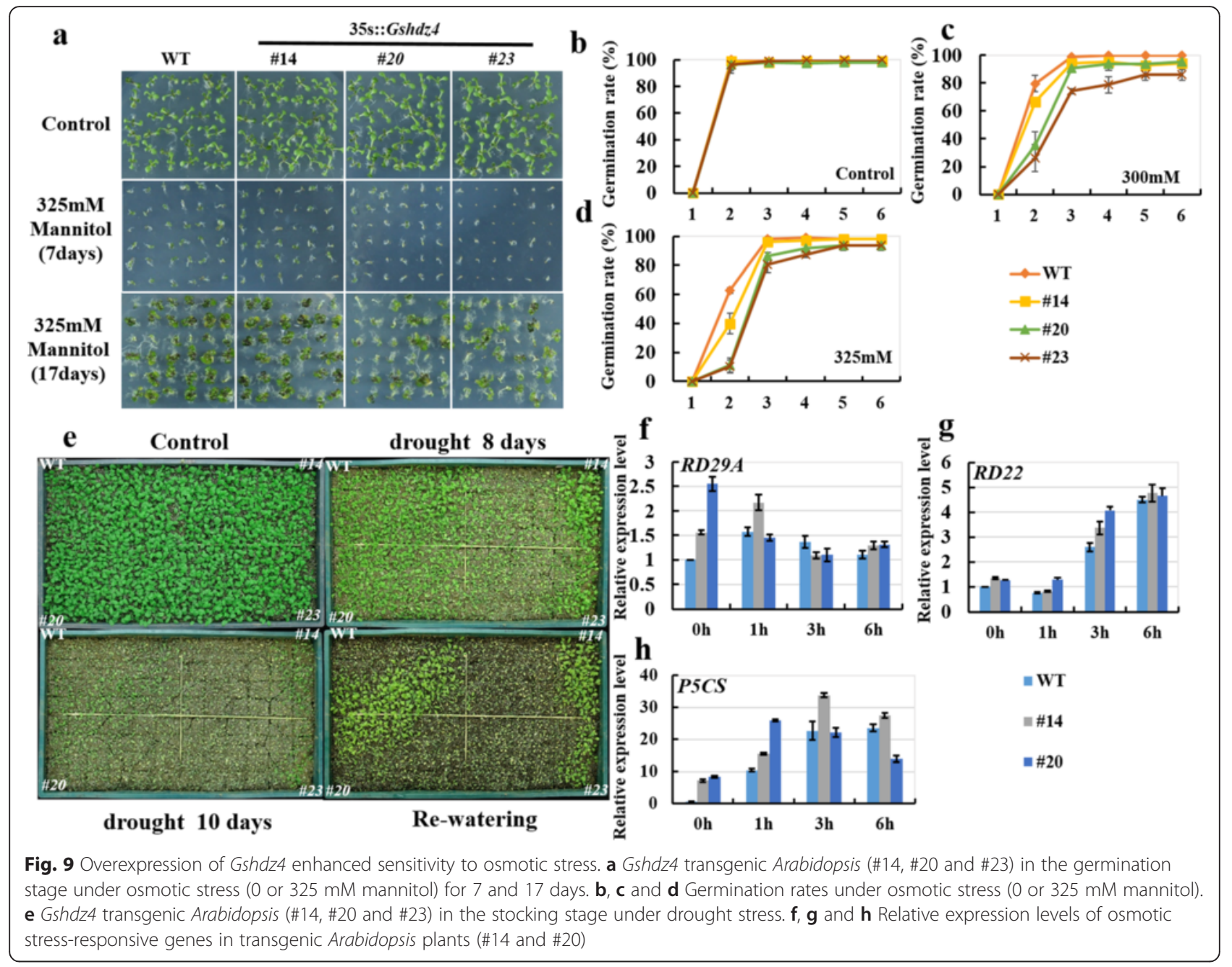

to $\mathrm{KHCO}_{3}$, but there was no obvious phenotype difference observed in $\mathrm{KOH}$ stress treatment at germination and seedling stages. Previous study suggests that under alkaline stress, plants might may activate much complicated responsive mechanisms in comparison with the plants under other stresses [2]. This may be led by the multiple toxicities of alkali stress, such as high $\mathrm{pH}$, $\mathrm{HCO}_{3}^{-}, \mathrm{CO}_{3}^{2-}$, and concomitant $\mathrm{Na}^{+}$in $\mathrm{NaHCO}_{3}$ solution $[38,39]$. As we still don't know the exact function of Gshdz4 in regulating alkaline stress, the further investigation is needed to dissect the mechanisms.

Our Gshdz4 transgenic Arabidopsis showed not only phenotypically increased tolerance to $\mathrm{HCO}_{3}{ }^{-}$stress, but also transcriptionally increased expression levels of stressresponsive genes, including $N A D P-M E, K I N 1, R D 29 B$ and $H^{+}$-Ppase. Most genes modulated by bicarbonate are involved in metabolism, transcription and signaling transduction [40]. In the previous studies, $V$ - $H^{+}$-PPase, PEPcase and NADP-ME were reported to be induced by bicarbonate stress $[41,42]$. These enzymes play significant roles in intracellular $\mathrm{pH}$ regulation, assisting plant cells in dealing with the potential acidification of the cytoplasm under environmental alkaline stress condition. Gshdz4 may modulate the process of bicarbonate stress tolerance through inducing the high expression of bicarbonate defense genes, such as NADP-ME and $H^{+}$-Ppase directly. KIN1 and RD29B are mainly induced by abscisic acid (ABA), cold and drought [43, 44]. The accumulation of these proteins contributes to adjusting physiological conditions in plant cells [45]. The ability of Gshdz4 to upregulate KIN1 and RD29B indicates that it may act as an important regulator in the process of bicarbonate resistance and participates in coordinating the expression of plant defense genes. However, growth regulation of plants in response to environmental stresses is extremely flexible and complex, and the complete molecular basis should be investigated further.

In order to defend the oxidative stresses, redox (oxidation-reduction)-related genes also play a key role in environmental stresses resistance, which can scavenge the reactive oxygen and then master the redox balance $[2,23,46,47]$. At the cellular level, alkaline stress causes 
the production of active $\mathrm{O}_{2}$ species, which result in the peroxidation of membrane lipid. MDA is an end product of lipid peroxidation. Our data showed that lower MDA content and higher POD activity existed in the transgenic plants than in the WT (Fig. 6f, g), implying that the degree of membrane injury of transgenic plants was less than that of WT, consistent with the enhanced alkaline tolerance phenotype of transgenic Arabidopsis.

Similarly, the transgenic plants showed greater tolerance to bicarbonate stress than the WT (Fig. 6d). In the absence of $\mathrm{NaHCO}_{3}$, there was almost no difference no matter in growth of plants or in contents of total chlorophyll among the WT and three transgenic lines. However, after supplement with $125 \mathrm{mM} \mathrm{NaHCO} 3$, the total chlorophyll contents in WT plants decreased more than in transgenic plants (Fig. 6e). These results suggested that overexpression of Gshdz4 reduced chlorophyll degradation and enhanced tolerance to bicarbonate in transgenic plants. However, the exact mechanism of Gshdz4 in this aspect is still unknown.

To our surprise, the negative regulation of drought stress by Gshdz4 was observed. Phenotypic experiments at both germination and seedling stages revealed that transgenic plants showed more sensitivity to osmotic stress than the WT, implying that Gshdz4 regulates alkaline and osmotic stresses by different ways. To better understand the molecular basis of the impaired drought stress tolerance of the Gshdz4 transgenic plants, the expression patterns of the stress-related genes, such as P5CS, RD22 and RD29A, were investigated. These genes have been used as convenient markers for monitoring the ABA and stress-responsive pathways in plants [48]. However, there was no significant difference observed in the experiment, implying that Gshdz4 may not regulate response to drought stress via these genes.

\section{Conclusions}

We identified a novel HD-ZIP I gene, Gshdz4, from Glycine soja. Gshdz4 encodes a protein with conserved homeodomain-leucine zipper domain and acts as a positive regulator in alkaline (bicarbonate) stress in Arabidopsis. The overexpression of Gshdz4 displays a significant tolerance to $\mathrm{NaHCO}_{3}$ stress in the seed germination, seedling growth and stocking stage. Moreover, transgenic lines showing tolerance to $\mathrm{KHCO}_{3}$ but not to $\mathrm{KOH}$ indicated that Gshdz4 may play an important role in bicarbonate tolerance pathway. What is more interesting is that the overexpression of Gshdz4 showed sensitivity to osmotic stress and did not regulate the marker genes in osmotic stress-relative pathways. Our studies broaden our knowledge level about HD-ZIP I family gene functions, and complement the evidences which was developed in previous studies.

\section{Methods}

\section{Plant materials and growing condition}

The seeds of G. soja G07256 were gained from Jilin Academy of Agricultural Sciences which is located in Changchun, China. At the first of the process, we treated the seeds with sulfuric acid (98\%) for $10 \mathrm{~min}$, washed them for 5 times using sterile water after which they were kept in darkness with a little sterile water for at least 2 days to facilitate germination. Following that, we transferred the seedlings to make them grow on $1 / 4$ Hoagland solution for 19 days at $24-26^{\circ} \mathrm{C}$ and in $16 \mathrm{~h} \mathrm{light} / 8 \mathrm{~h}$ dark cycles. To study the tissue-specific expression of Gshdz4, the samples of young leaves, old leaves, young stems, old stems, epicotyls, hypocotyls, roots and flowers were taken from the wild soybean plants. To detect the expression profile of Gshdz4 gene under alkali-stress treatment, the 19day seedlings were kept in $1 / 4$ Hoagland liquid mediums containing $50 \mathrm{mM} \mathrm{NaHCO} 3$ (pH 8.5) for $0,1,3,6,12$ or $24 \mathrm{~h}$, respectively. All the samples were stored at $-80{ }^{\circ} \mathrm{C}$ after snap-frozen in liquid nitrogen.

Arabidopsis thaliana Columbia (Col-0) was used as the wild-type plant for transformation. The WT and transgenic seeds of Arabidopsis were grown in a greenhouse under the controlled environmental conditions $\left(21-23{ }^{\circ} \mathrm{C}, 100 \mu \mathrm{mol}\right.$ photons $\mathrm{m}^{-2} \mathrm{~s}^{-1}, 60 \%$ relative humidity, $16 \mathrm{~h}$ light $/ 8 \mathrm{~h}$ dark cycles). The samples were collected as mentioned above.

\section{Identification and bioinformatics analysis of Gshdz4}

The cDNA (full-length) of Gshdz4 was obtained by homologous cloning. The total RNA of G. soja G07256 was isolated from leaves by EasyPure RNA Kit (Transgen Biotech) and was converted to CDNA by reverse transcription using GoldScript cDNA Synthesis Kit (Invitrogen). The fulllength cDNA of Gshdz4 was amplified using gene-specific primers: 5'-AAGCCAGTGAAGAGCGAGCGAGA-3' and 5'-CTACCATTTCGGGTCAAATTGCAAA-3'. The PCR product was cloned into the pEASY-Blunt Zero Cloning Vector (Transgen Biotech) and subjected to sequencing. Sequence alignments were performed using DNAMAN. Phylogenetic analysis was carried out with MEGA 4.0. Similarity of sequences was verified by the BLASTP at NCBI online (http://blast.ncbi.nlm.nih.gov/Blast.cgi). Homology searches were done by Phytozome (http://www.phytozome.net/soybean).

\section{Subcellular localization analysis}

Gshdz4 CDS was amplified from pEASY-Blunt Zero Cloning Vector by PCR with BamHI and SpeI linker primers: 5' - TAATATGGATCCCATGAATCATCGACCA CCTTTC-3' and 5'-AATTATACTAGTGCATATACAGATTAATCCATTCCATG-3'. The full-length Gshdz4 gene was cloned into pBSK-eGFP vector. The recombinant Gshdz4-green fluorescent protein (GFP) plasmid was 
bombarded into epidermal cells of onion with particle bombardment. Analysis of GFP was performed as mentioned previously [49].

\section{Transcriptional activation analysis}

The full-length CDS (coding sequence) of Gshdz4 was amplified by PCR using NdeI and SalI linker primers: 5' TAATATCATATGGGATGAATCATCGACCACCTTTC$3^{\prime}$ and $5^{\prime}$ - AATTATGTCGACGTTACATATACAGATTA ATCCATTCCATG-3'. The gene was introduced into pGBKT7 vector. The pGBKT7-GsDREB (positive control) and the pGBKT7 (negative control) were transformed into yeast strain AH109. Transcription-activation activity was analyzed following the protocols mentioned previously [50].

\section{Generation of transgenic Arabidopsis thaliana}

The pCAMBIA330035Su-Gshdz4 plasmid was constructed by amplifying the full-length CDS of Gshdz4 from pEASY-Blunt Zero Cloning-Gshdz4 by PCR using primers: 5'-GGCTTAAUAAGCCAGTGAAGAGCGAG CGAGA-3' and 5'-GGTTTAAUCTACCATTTCGGGTCAAATTGCAAA-3'. The underlined nucleotide sequences indicate $P a c$ I restriction enzyme cutting site. The gene was cloned into a USER (uracil-specific excision reagent) vector pCAMBIA330035Su as describe previously [51]. The resulted vector was mobilized into Agrobacterium tumefaciens strainGV3101. Plant transformation was performed by using the floral dipping method [52]. The transgenic plants from $T_{1}$ generation were screened on $1 / 2$ MS solid mediums containing $25 \mathrm{mg} \mathrm{L}^{-1}$ glufosinate. The seeds from each $\mathrm{T}_{1}$ plant were individually collected. The selected $T_{2}$ plants were sowed, and the transgenic lines were confirmed by RT-PCR assay (Fig. 5a). The homozygous overexpression lines (\#14, \#20 and \#23) were obtained by $\mathrm{PCR}$ and the progeny segregation in $\mathrm{T}_{3}$ generation.

\section{Phenotypic analysis of transgenic Arabidopsis under the treatments of $\mathrm{NaHCO}_{3}, \mathrm{KHCO}_{3}, \mathrm{KOH}$ and drought}

We selected three $T_{3}$ homozygous overexpression lines (\# 14, \# 20, and \# 23) to study their resistance to various stresses. The WT and homozygous transgenic Arabidopsis seeds were sterilized and were sown on $0.5 \times$ MS agar medium, which were supplemented with $0,6,7 \mathrm{mM}$ $\mathrm{NaHCO}_{3}$, or $6 \mathrm{mM} \mathrm{KHCO}$ or $325 \mathrm{mM}$ mannitol or $\mathrm{KOH}$ to make the medium at $\mathrm{pH} 7.5$ or $\mathrm{pH} 8.2$. For root length and fresh weight assays, the seeds were germinated on $0.5 \times$ MS agar medium for 7 days and then were transferred to fresh medium supplemented with 0,6 , $8 \mathrm{mM} \mathrm{NaHCO}, 6 \mathrm{mM} \mathrm{KHCO} 3,325 \mathrm{mM}$ mannitol, or $\mathrm{KOH}$ to adjust the medium to $\mathrm{pH} 7.5, \mathrm{pH}$ 8.2. The seedlings were grown on the medium for 12-14 days and the lengths of roots were measured. All experiments were performed 3 completely independent biological replicates at least. All statistical analysis was done with EXCEL2010 (Microsoft, http://www.microsoftstore.com.cn/).

To study of Gshdz4 transgenic Arabidopsis phenotypes, the seeds from WT and transgenic plants were sown in the soil supplemented with appropriate vermiculite and the 3-week-old plants were treated by water containing either 0 or $125 \mathrm{mM} \mathrm{NaHCO} 3$ every 3 days for 15 days. To detect drought resistance at the adult stages, water was withheld for 10 days from 21-day-old plants.

The data were analyzed by EXCEL 2010 and the results from one representative experiment were shown (all assays were repeated at least three times).

\section{Quantitative real-time PCR analysis of Gshdz4 and stress response genes}

Total RNA was extracted from G. soja or Arabidopsis seedlings by using EasyPure RNA kit (Transgen Biotech), and cDNA synthesis was performed using the GoldScript cDNA Synthesis kit (Invitrogen, Carlsbad, CA, USA). cDNA quality was assessed by PCR using specific primers for GAPDH (glyceraldehyde-3-phosphate dehydrogenase, accession no. DQ355800) to exclude genomic DNA contamination. The qRT-PCR was performed in 96-well $(25 \mu \mathrm{L})$ format using the SYBR Premix ExTaq ${ }^{\text {Tix }}$ II Mix (TaKaRa, Shiga, Japan) on an ABI 7500 sequence detection system (Applied Biosystems, USA). One microliter of each synthesized cDNA (diluted 1:5) was used as template. Amplification of GAPDH in G. soja or ACTIN2 in Arabidopsis was used as controls. Expression levels for all candidate genes were determined using the $2^{-\Delta \Delta C T}$ method. The relative transcript levels were calculated and normalized as described previously [53]. cDNA from three independent plants were treated as three biological replicates and each cDNA sample was repeated three times as three technical replicates, and the results from one representative experiment are shown.

\section{Chlorophyll content, POD activity and MDA content}

For measurement of chlorophyll content, $1 \mathrm{~g}$ of fresh leaf samples from transgenic lines and WT plants were quickly homogenized in $0.5 \mathrm{~mL}$ of $100 \%$ acetone followed by addition of $1 \mathrm{~mL}$ of $80 \%$ acetone to determine the chlorophyll contents according to the method described previously [54]. The absorbance values of chlorophyll $a$ and chlorophyll $b$ were respectively measured at 663 and $645 \mathrm{~nm}$ using an ultraviolet spectrophotometer (UV-2550, Shimadzu, Tokyo, Japan).

To assess peroxidase (POD) activity, fresh leaf segments from the same locus of each plant were respectively homogenized in $0.05 \mathrm{~mol} / \mathrm{L}$ phosphate buffer $(\mathrm{pH}$ 5.5). The homogenates were transferred into centrifuge tubes and were centrifuged at $4000 \mathrm{rpm}$ for $10 \mathrm{~min}$. The absorbance at $470 \mathrm{~nm}$ was measured using 
an ultraviolet spectrophotometer (UV-2550, Shimadzu, Tokyo, Japan).

The MDA content was determined using the thiobarbituric acid (TBA) protocol $[55,56]$. Equal amount of leaves from transgenic lines and WT plants were frozen by liquid nitrogen and grinded into powdery in $1 \mathrm{~mL}$ $5 \%$ trichloroacetic (TCA). The homogenates were centrifuged at 15,000 rpm for $20 \mathrm{~min}$ and the supernatants were taken to mix well with $800 \mu \mathrm{L}$ of $0.5 \%$ TBA. The reaction mixtures were heated at $95{ }^{\circ} \mathrm{C}$ for $30 \mathrm{~min}$ and were cooled on ice and then were centrifuged at $15,000 \mathrm{rpm}$ for $20 \mathrm{~min}$ again. The absorbance at 450 , 532 , and $600 \mathrm{~nm}$ was respectively measured using an ultraviolet spectrophotometer (UV-2550, Shimadzu, Tokyo, Japan).

\section{Additional files}

Additional file 1: Figure S1. Germination rates under $\mathrm{NaHCO}_{3}$ (0, 6 or 7 mM) stress. (TIF 568 kb)

Additional file 2: Figure S3. No difference in growth between the overexpression lines and WT plants in the normal condition. (TIF $3134 \mathrm{~kb}$ )

Additional file 3: Figure S2. Germination rates under $\mathrm{KHCO}_{3}$ ( 0 or $6 \mathrm{mM}$ ) and $\mathrm{KOH}(\mathrm{pH} 7.5$ or pH8.2) stresses. (TIF $549 \mathrm{~kb}$ )

\section{Acknowledgements}

We would like to thank Prof. Guanzheng Qu for the service of confocal laser-scanning microscope in Northeast Forestry University.

\section{Funding}

This work was supported by the National Natural Science Foundation of China (31171578), and the Technological Innovation Team Building Program of the College of Heilongjiang Province (2011TD005) and NEAU starting grant to XD.

\section{Availability of data and materials}

The data sets supporting the results of this article are included within the article and its additional files. The sequence of Gshdz4 has submitted to GenBank and the accession No. is KX257484.

\section{Authors' contributions}

Experiment designation: $L C, Y Z, X D$. Experiment performance and data analyses: LC, YY, HD, QL, CC, PZ, RC, XBD. Manuscript drafting: LC, XD. All authors read and approved the final manuscript.

\section{Competing interests}

The authors declare that they have no competing interests.

\section{Consent for publication}

Not applicable.

\section{Ethics approval and consent to participate}

Not applicable.

Received: 21 May 2016 Accepted: 15 August 2016

Published online: 24 August 2016

\section{References}

1. Ashraf M, Akram NA. Improving salinity tolerance of plants through conventional breeding and genetic engineering: An analytical comparison. Biotechnol Adv. 2009;27(6):744-52.

2. DuanMu H, Wang Y, Bai X, Cheng S, Deyholos MK, Wong GK, Li D, Zhu D, Li $R, Y u Y$, et al. Wild soybean roots depend on specific transcription factors and oxidation reduction related genesin response to alkaline stress. Funct Integr Genomics. 2015;15:651-60.

3. Ariel FD, Manavella PA, Dezar CA, Chan RL. The true story of the HD-Zip family. Trends Plant Sci. 2007;12(9):419-26.

4. Zhu N, Cheng S, Liu X, Du H, Dai M, Zhou DX, Yang W, Zhao Y. The R2R3-type MYB gene OsMYB91 has a function in coordinating plant growth and salt stress tolerance in rice. Plant Science. 2015;236:146-56.

5. Luo X, Bai X, Sun X, Zhu D, Liu B, Ji W, Cai H, Cao L, Wu J, Hu M, et al. Expression of wild soybean WRKY20 in Arabidopsis enhances drought tolerance and regulates ABA signalling. J Exp Bot. 2013;64(8): 2155-69.

6. Puranik S, Sahu PP, Srivastava PS, Prasad M. NAC proteins: regulation and role in stress tolerance. Trends Plant Sci. 2012;17(6):369-81.

7. Liu C, Mao B, Ou S, Wang W, Liu L, Wu Y, Chu C, Wang X. OsbZIP71, a bZIP transcription factor, confers salinity and drought tolerance in rice. Plant Mol Biol. 2014;84(1-2):19-36

8. Luo X, Cui N, Zhu Y, Cao L, Zhai H, Cai H, Ji W, Wang X, Zhu D, Li Y, et al. Over-expression of GsZFP1, an ABA-responsive C2H2-type zinc finger protein lacking a QALGGH motif, reduces ABA sensitivity and decreases stomata size. J Plant Physiol. 2012;169(12):1192-202.

9. Zhu D, Bai X, Chen C, Chen Q, Cai H, Li Y, Ji W, Zhai H, Lv D, Luo X, et al. GSTIFY10, a novel positive regulator of plant tolerance to bicarbonate stress and a repressor of jasmonate signaling. Plant Mol Biol. 2011;77(3):285-97.

10. Chen X, Chen Z, Zhao H, Zhao Y, Cheng B, Xiang Y. Genome-Wide Analysis of Soybean HD-Zip Gene Family and Expression Profiling under Salinity and Drought Treatments. PLoS One. 2014;9(2):e87156.

11. Mukherjee K, Burglin TR. MEKHLA, a novel domain with similarity to PAS domains, is fused to plant homeodomain-leucine zipper III proteins. Plant Physiol. 2006;140(4):1142-50.

12. Landschulz WH, Johnson PF, McKnight SL. The leucine zipper: a hypothetical structure common to a new class of DNA binding proteins. Science. 1988;240(4860):1759-64.

13. Zhang S, Haider I, Kohlen W, Jiang L, Bouwmeester H, Meijer AH, Schluepmann H, Liu CM, Ouwerkerk PB. Function of the HD-Zip I gene Oshox22 in ABA-mediumted drought and salt tolerances in rice. Plant Mol Biol. 2012;80(6):571-85

14. Valdes AE, Overnas $E$, Johansson $H$, Rada-Iglesias A, Engstrom P. The homeodomain-leucine zipper (HD-Zip) class I transcription factors ATHB7 and ATHB12 modulate abscisic acid signalling by regulating protein phosphatase $2 \mathrm{C}$ and abscisic acid receptor gene activities. Plant Mol Biol. 2012;80(4-5):405-18.

15. Zhao $Y, M a ~ Q$, Jin $X$, Peng $X$, Liu J, Deng L, Yan $H$, Sheng $L$, Jiang $H$, Cheng B. A novel maize homeodomain-leucine zipper (HD-Zip) I gene, Zmhdz10, positively regulates drought and salt tolerance in both rice and Arabidopsis. Plant Cell Physiol. 2014;55(6):1142-56.

16. Ariel F, Diet A, Verdenaud M, Gruber V, Frugier F, Chan R, Crespi M. Environmental regulation of lateral root emergence in Medicago truncatula requires the HD-Zip I transcription factor HB1. Plant Cell. 2010;22(7):2171-83.

17. Yang $X$, Hajiboland R, Romheld V. Bicarbonate had greater effects than high $\mathrm{pH}$ on inhibiting root growth of zinc-inefficient rice genotype. J Plant Nutr. 2003;26(2):399-415.

18. Belamkar V, Weeks NT, Bharti AK, Farmer AD, Graham MA, Cannon SB. Comprehensive characterization and RNA-Seq profiling of the HD-Zip transcription factor family in soybean (Glycine max) during dehydration and salt stress. BMC Genomics. 2014;15:950.

19. Sun M, Sun X, Zhao Y, Zhao C, DuanMu H, Yu Y, Ji W, Zhu Y. Ectopic Expression of GsPPCK3 and SCMRP in Medicago sativa Enhances Plant Alkaline Stress Tolerance and Methionine Content. PLoS One. 2014;9(2):e89578.

20. Kotchoni SO, Kuhns C, Ditzer A, Kirch H-H, Bartels D. Over-expression of different aldehyde dehydrogenase genes in Arabidopsis thaliana confers tolerance to abiotic stress and protects plants against lipid peroxidation and oxidative stress. Plant, Cell Environ. 2006:29(6):1033-48.

21. Weber $H$, Chételat A, Reymond P, Farmer EE. Selective and powerful stress gene expression inArabidopsisin response to malondialdehyde. Plant J. 2004;37(6):877-88

22. Huang XS, Liu JH, Chen XJ. Overexpression of PtrABF gene, a bZIP transcription factor isolated from Poncirus trifoliata, enhances dehydration and drought tolerance in tobacco via scavenging ROS and modulating expression of stress-responsive genes. BMC Plant Biol. 2010;10:230.

23. Jaspers $P$, Kangasjarvi J. Reactive oxygen species in abiotic stress signaling. Physiol Plant. 2010;138(4):405-13. 
24. Zhao Y, Xu T, Shen CY, Xu GH, Chen SX, Song LZ, Li MJ, Wang LL, Zhu Y, Lv WT, et al. Identification of a Retroelement from the Resurrection Plant Boea hygrometrica That Confers Osmotic and Alkaline Tolerance in Arabidopsis thaliana. PLoS One. 2014;9(5):e98098.

25. Guo R, Shi LX, Yang YF. Germination, growth, osmotic adjustment and ionic balance of wheat in response to saline and alkaline stresses. Soil Sci Plant Nutr. 2009;55(5):667-79.

26. Ge Y, Li Y, Zhu YM, Bai X, Lv DK, Guo D, Ji W, Cai H. Global transcriptome profiling of wild soybean (Glycine soja) roots under $\mathrm{NaHCO} 3$ treatment. BMC Plant Biol. 2010;10:153.

27. Rueda EC, Dezar CA, Gonzalez DH, Chan RL. Hahb-10, a sunflower homeobox-leucine zipper gene, is regulated by light quality and quantity, and promotes early flowering when expressed in Arabidopsis. Plant Cell Physiol. 2005;46(12):1954-63.

28. Sessa G, Carabelli M, Sassi M, Ciolfi A, Possenti M, Mittempergher F, Becker J, Morelli G, Ruberti I. A dynamic balance between gene activation and repression regulates the shade avoidance response in Arabidopsis. Genes Dev. 2005;19(23):2811-5.

29. Morelli G, Ruberti I. Light and shade in the photocontrol of Arabidopsis growth. Trends Plant Sci. 2002;7(9):399-404.

30. Morelli G, Ruberti I. Shade avoidance responses. Driving auxin along lateral routes. Plant Physiol. 2000;122(3):621-6.

31. Sawa S, Ohgishi M, Goda H, Higuchi K, Shimada Y, Yoshida S, Koshiba T. The HAT2 gene, a member of the HD-Zip gene family, isolated as an auxin inducible gene by DNA microarray screening, affects auxin response in Arabidopsis. Plant J. 2002;32(6):1011-22.

32. Delarue M, Prinsen E, Onckelen HV, Caboche M, Bellini C. Sur2 mutations of Arabidopsis thaliana define a new locus involved in the control of auxin homeostasis. Plant J. 1998;14(5):603-11.

33. Manavella PA, Arce AL, Dezar CA, Bitton F, Renou JP, Crespi M, Chan RL. Crosstalk between ethylene and drought signalling pathways is mediumted by the sunflower Hahb-4 transcription factor. Plant J. 2006:48(1):125-37.

34. Zhao Y, Ma Q, Jin X, Peng X, Liu J, Deng L, Yan H, Sheng L, Jiang H, Cheng B. A Novel Maize Homeodomain-leucine Zipper (HD-Zip) I Gene, Zmhdz10, Positively Regulates Drought and Salt tolerance in both Rice and Arabidopsis. Plant Cell Physiol. 2014;55(6):1142-56. doi:10.1093/pcp/pcu054.

35. Shan CM, Shangguan XX, Zhao B, Zhang XF, Chao LM, Yang CQ, Wang $L$, Zhu HY, Zeng YD, Guo WZ, et al. Control of cotton fibre elongation by a homeodomain transcription factor GhHOX3. Nat Commun. 2014;5:5519.

36. Hur YS, Um JH, Kim S, Kim K, Park HJ, Lim JS, Kim WY, Jun SE, Yoon EK, Lim J, et al. Arabidopsis thaliana homeobox 12 (ATHB12), a homeodomainleucine zipper protein, regulates leaf growth by promoting cell expansion and endoreduplication. New Phytol. 2015;205(1):316-28.

37. De Smet I, Lau S, Ehrismann JS, Axiotis I, Kolb M, Kientz M, Weijers D, Jurgens $\mathrm{G}$. Transcriptional repression of BODENLOS by HD-ZIP transcription factor HB5 in Arabidopsis thaliana. J Exp Bot. 2013;64(10):3009-19.

38. Koropatkin NM, Koppenaal DW, Pakrasi HB, Smith TJ. The structure of a cyanobacterial bicarbonate transport protein, CmpA. J Biol Chem. 2007; 282(4):2606-14.

39. Wang Y, Yang C, Liu G, Jiang J. Development of a CDNA microarray to identify gene expression of Puccinellia tenuiflora under saline-alkali stress. Plant Physiol Biochem. 2007;45(8):567-76.

40. Alhendawi RA, Römheld V, Kirkby EA, Marschner H. Influence of increasing bicarbonate concentrations on plant growth, organic acid accumulation in roots and iron uptake by barley, sorghum, and maize. J Plant Nutr. 1997;20(12):1731-53.

41. Fushimi T, Umeda M, Shimazaki T, Kato A, Toriyama K, Uchimiya $\mathrm{H}$. Nucleotide sequence of a rice cDNA similar to a maize NADP-dependent malic enzyme. Plant Mol Biol. 1994;24(6):965-7.

42. Liu S, Zhang $X$, Chen $Y$. "Is the "plant intracellular pH regulation system" a tolerance mechanism adapting to environmental stress? Molecular". Plant Breed. 2004;2(2):179-86.

43. Kurkela S, Franck M. Cloning and characterization of a cold- and ABAinducible Arabidopsis gene. Plant Mol Biol. 1990;15(1):137-44

44. Nordin K, Vahala T, Palva ET. Differential expression of two related, lowtemperature-induced genes in Arabidopsis thaliana (L.) Heynh. Plant Mol Biol. 1993;21(4):641-53

45. Tardieu F, Davies WJ. Stomatal response to abscisic Acid is a function of current plant water status. Plant Physiol. 1992;98(2):540-5.

46. Gao CQ, Wang YC, Liu GF, Yang CP, Jiang J, Li HY. Expression profiling of salinity-alkali stress responses by large-scale expressed sequence tag analysis in Tamarix hispid. Plant Mol Biol. 2008;66(3):245-58.
47. Miller G, Shulaev V, Mittler R. Reactive oxygen signaling and abiotic stress. Physiol Plant. 2008:133(3):481-9.

48. Pandey GK, Cheong YH, Kim KN, Grant JJ, Li L, Hung W, D’Angelo C, Weinl S, Kudla J, Luan S. The calcium sensor calcineurin B-like 9 modulates abscisic acid sensitivity and biosynthesis in Arabidopsis. Plant Cell. 2004;16(7):1912-24.

49. Gong Z, Morales-Ruiz T, Ariza RR, Roldan-Arjona T, David L, Zhu JK. ROS1, a repressor of transcriptional gene silencing in Arabidopsis, encodes a DNA glycosylase/lyase. Cell. 2002;111(6):803-14.

50. Yang L, Ji W, Zhu Y, Gao P, Li Y, Cai H, Bai X, Guo D. GsCBRLK, a calcium/ calmodulin-binding receptor-like kinase, is a positive regulator of plant tolerance to salt and ABA stress. J Exp Bot. 2010:61(9):2519-33.

51. Nour-Eldin HH, Hansen BG, Norholm MH, Jensen JK, Halkier BA. Advancing uracil-excision based cloning towards an ideal technique for cloning PCR fragments. Nucleic Acids Res. 2006;34(18):e122.

52. Clough SJ, Bent AF. Floral dip: a simplified method for Agrobacteriummediumted transformation of Arabidopsis thaliana. Plant J. 1998;16(6):735-43.

53. Willems $E$, Leyns L, Vandesompele J. Standardization of real-time PCR gene expression data from independent biological replicates. Anal Biochem. 2008;379(1):127-9.

54. Lichtenthaler HK, Wellburn AR. Determinations of total carotenoids and chlorophylls a and b of leaf extracts in different. Biochem Soc Trans. 1983;11:592.

55. Dong W, Wang M, Xu F, Quan T, Peng K, Xiao L, Xia G. Wheat oxophytodienoate reductase gene TaOPR1 confers salinity tolerance via enhancement of abscisic acid signaling and reactive oxygen species scavenging. Plant Physiol. 2013;161(3):1217-28.

56. Xu J, Duan $X$, Yang J, Beeching JR, Zhang $P$. Enhanced reactive oxygen species scavenging by overproduction of superoxide dismutase and catalase delays postharvest physiological deterioration of cassava storage roots. Plant Physiol. 2013;161(3):1517-28.

\section{Submit your next manuscript to BioMed Central and we will help you at every step:}

- We accept pre-submission inquiries

- Our selector tool helps you to find the most relevant journal

- We provide round the clock customer support

- Convenient online submission

- Thorough peer review

- Inclusion in PubMed and all major indexing services

- Maximum visibility for your research

Submit your manuscript at www.biomedcentral.com/submit
) Biomed Central 